\title{
Sedation for Brain Magnetic Resonance Imaging in Preterm Infants: Using Propofol under Anesthesiologist Supervision
}

Yea Seul Han, $\mathrm{MD}^{1}$, Hyun Ho Kim, MD, $\mathrm{PhD}^{2}$, Hye Seon Kim, $\mathrm{MD}^{2}$, Mi Sun Yang, $\mathrm{MD}^{2}$, So Yoon Ahn, $\mathrm{MD}, \mathrm{PhD}^{2}$, Se In Sung, $\mathrm{MD}, \mathrm{PhD}^{2}$, Yun Sil Chang, $\mathrm{MD}, \mathrm{PhD}^{2}$, and Won Soon Park, $\mathrm{MD}, \mathrm{PhD}^{2}$

${ }^{1}$ Department of Pediatrics, Hallym University Kangnam Sacred Heart Hospital, Seoul, Korea

${ }^{2}$ Department of Pediatrics, Samsung Medical Center, Sungkyunkwan University School of Medicine, Seoul, Korea

\section{ABSTRACT}

Purpose: We aimed to compare two different sedation protocols for brain magnetic resonance imaging (MRI) in preterm infants. One protocol used chloral hydrate (CH) with monitoring conducted by non-anesthesiologists, and the other used a continuous infusion of propofol (PF) with monitoring by anesthesiologists.

Methods: A total of 250 preterm infants born between January 2011 and December 2015 who received brain MRI during hospitalization in our neonatal intensive care unit (NICU) were included in this retrospective study. In period 1, sedation for brain MRI was done using a single dose or multiple doses of $\mathrm{CH}$ with monitoring conducted by NICU medical staff. In period 2, an anesthesiologist prescribed a continuous infusion of PF and titrated the dosage for minimal and adequate sedation. Data on the adverse events, including desaturation and bradycardia, were collected and compared between periods 1 and 2 .

Results: Despite similar gestational ages of the patients in periods 1 and 2, the infants in period 1 showed a higher risk of developing bradycardia after sedation compared to those in period 2 (30.2\% vs. $14.8 \%$; an adjusted odds ratio of 2.35 ; $95 \%$ confidence interval of 1.12 to 4.91). Infants who had an adverse event after sedation had a lower gestational age and corrected age at the time of MRI (26.8 weeks vs. 27.9 weeks, $P=0.004$; 37.3 weeks vs. 38.3 weeks, $P=0.023$ ). The duration of MRI was significantly longer in infants that had an adverse event than those that did not (70.9 minutes vs. 64.3 minutes). After adjusting for various clinical factors, lower gestational age, lower corrected age at the time of MRI, and period 1 increased the risk of developing adverse events after sedation for MRI.

Conclusion: The use of a continuous PF infusion with dose titration and monitoring by an anesthesiologist is safe and feasible as a sedation protocol for brain MRI in prematurely born infants.

Key Words: Magnetic resonance imaging; Anesthesiologists; Propofol; Infant, premature
Received: 22 April 2020

Revised: 22 June 2020

Accepted: 27 June 2020

Correspondence to: Se In Sung, MD, $\mathrm{PhD}$

Department of Pediatrics, Samsung Medical Center, Sungkyunkwan University School of Medicine, 81 Irwonro, Gangnam-gu, Seoul 06351, Korea

Tel: +82-2-3410-1775

Fax: +82-2-3410-0043

E-mail: sein.sung@samsung.com

\footnotetext{
Copyright(c)

By Korean Society of Neonatology.

All right reserved.

This is an Open-Access article distributed under the terms of the Creative Commons Attribution Non-Commercial License (http:// creativecommons.org/licenses/by-nc/4.0), which permits unrestricted non-commercial use, distribution, and reproduction in any medium, provided the original work is pro perly cited.
} 
서론

뇌 자기공명영상(magnetic resonance imaging, MRI)은 미숙아나 고위험 신생아의 뇌 손상을 평가하여 환자의 상태를 이해하는 데 도움이 될 수 있으나 체동 아티팩트(motion artifact)에 민감하기 때 문에 좋은 영상을 얻기 위해서는 적절한 진정요법이 필요할 수 있 다 ${ }^{1,2)}$. 신생아의 진정 치료에 사용되는 약물은 일반적으로 chloral hydrate (CH; Pocral syrup, Hanlim, Seoul, Korea), 미다졸람(midazolam), 프로포폴(propofol, 이하 PF) 등이 사용 가능하며 ${ }^{3)}$, 그 중 $\mathrm{CH}$ 는 경구투여가 가능하고, 심각한 부작용이 적어 소아에서 많이 사용되어 왔으나 진정 실패로 반복적 약물투여가 필요할 수 있다 ${ }^{4-6)}$. 이에 반해 $\mathrm{PF}$ 는 호흡억제 작용이 강하지만 진정 유도가 빠르고(1-2 분) 진정 효과가 좋으며, 회복이 빠르므로 MRI실의 운영에 있어 시 간-비용 면의 이득을 가져올 수 있어 임상적으로 선호되는 약제이다 7-9). 그러나 대부분의 진정 약물이 소아 및 청소년보다 신생아의 체 내에 지속되는 시간이 길게 유지되어 호흡 및 심혈관계 억제가 유발 될 가능성이 높으므로 신생아 및 소아에서 진정마취검사를 시행할 시엔 호흡 및 순환기 상태를 안전하게 유지하면서 적절한 진정의 균 형을 유지하는 것이 중요하다 ${ }^{10,11}$.

최근에는 병원 차원에서 진정 전문인력을 배치하고 체계적인 소 아 진정의 프로토콜을 개발하려 노력하는 중이나, 현실적으로 신생 아, 특히 미숙아로 출생한 환자에서 진정요법을 시행할 시 비 마취 과 의사에 의해 이루어지는 경우가 많으며, 어떻게 시행하는 것이 이상적인지에 대한 연구가 부족한 실정이다 ${ }^{12,13)}$. 이에 신생아중환 자실에 입원 중인 미숙아 출생 영아의 뇌 MRI 촬영 시, 비 마취과 의 료진의 처방과 감시 하의 경관 $\mathrm{CH}$ 사용을 통한 진정요법과 소아 전 담 마취과 의사의 감독 하의 $\mathrm{PF}$ 연속주입을 통한 진정요법 간의 이 상반응 빈도를 비교하고, 이상반응 발생에 영향을 미칠 수 있는 위 험인자를 확인하고자 본 연구를 시행하였다.

\section{대상 및 방법}

\section{1. 연구대상}

본 연구는 2011년 1월부터 2015년 12월까지 본원 신생아중환자 실에 입원하여 뇌 MRI를 시행한 모든 미숙아(임신나이 37주 0일 미 만, 총 대상자 수 $=250$ )를 대상으로 차트 리뷰를 통해 후향적으로 분 석하였다. MRI 촬영 시기에 따라 1기(2011년 1월에서 2014년 12월, 169명)와 2기(2015년 1월에서 2015년 12월, 81명)로 구분하였다. 2016년 이후로는 신생아중환자실 입원 기간 중 MRI 촬영을 지양하 고 생후 12 개월 경 외래에서 선택적으로 MRI 촬영을 시행하였기 때 문에 연구 기간에서 제외하였다. 1 기에서는 MRI 촬영 시 신생아중 환자실 의사 및 비 마취과 의료인에 의해 $\mathrm{CH}$ 투여를 통해 진정이 이
루어졌으며, 2 기에서는 소아 전담 마취과 의사에 의해 $\mathrm{PF}$ 연속주입 이 이루어지며 모니터링되었다. 단, 1 기에서는 모든 미숙아에서 만 삭나이에 도달했을 때 일률적으로 뇌 MRI가 시행되었으나 2기에서 는 뇌 초음파검사에서 3-4단계 뇌실내출혈, 저산소성 허혈성 뇌병 증, 뇌 선천기형 등의 뇌질환이 의심, 동반되었을 때에만 선택적으 로 MRI를 시행하였다(Figure 1). 뇌질환 의심 등에 따른 MRI 촬영 여 부는 담당 임상의의 임상적 판단에 따라 결정하였다.

\section{2. 진정요법지침}

1 기에서는 신생아중환자실에서 $\mathrm{CH} 50 \mathrm{mg} / \mathrm{kg}$ 을 경관 투여하였 고, 초회 용량으로 진정 실패 시 20-30분 경과 후 동량을 한 차례 더 투여하였으며 ${ }^{14)}, \mathrm{MRI}$ 실에서 미다졸람 $0.1 \mathrm{mg} / \mathrm{kg}$ 를 최대 2회까지 정맥 내 투여하였다. 반면 2기에서는 MRI실에서 소아 전담 마취과 의사가 마취 유도를 위해 PF를 1-2 mg/kg 일시 투여 후 $10 \mu \mathrm{g} / \mathrm{kg} /$ $\min$ 의 용량으로 연속주입을 시작하였고, 진정 정도 및 활력징후를 감시하면서 개별 기준선으로부터 PF 주입속도를 10-50 $\mu \mathrm{g} / \mathrm{kg} / \mathrm{min}$ 범위 내에서 단계적으로 증가시켰으며, 진정이 이루어지는 최소 용 량으로 조정(titration)하였다. 1 기와 2기 모두 뇌 MRI 시행 전 4시간 의 금식을 시행하였고 ${ }^{15)}$, 전공의가 이동용 장비로 활력징후를 모니 터링하면서 MRI실로 이동하였다.

\section{3. 자료수집}

본원의 전자의무기록을 이용하여 각 대상의 인구학적 데이터 및 진정치료방법, 이상반응 발생 여부를 조사하였다. 진정요법 중에 발 생한 이상반응은 동행한 의사 및 $\mathrm{MRI}$ 실에서 기록한 진정 기록지를 통하여, 진정 치료가 끝난 후 발생한 이상반응은 담당 주치의 및 간 호사에 의해 기록된 임상기록을 통하여 확인하였다. 인구학적 데이 터는 출생 시 임신나이, 체중 및 외부출생 여부, 기관지폐이형성증, 뇌 기형 여부, 3-4단계의 뇌실내출혈 등을 조사하였으며, 이상반응

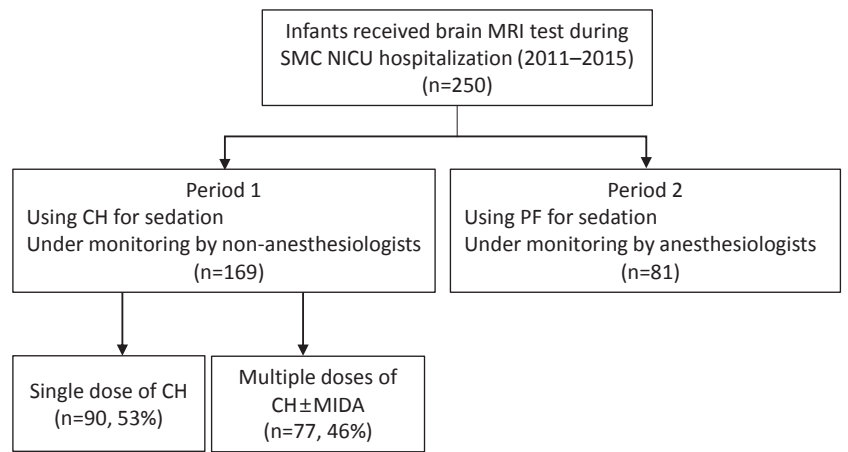

Figure 1. Study population. A total of 250 preterm infants born between 2011 to 2015 were included. Abbreviations: MRI, magnetic resonance imaging; SMC, Samsung Medical Center; NICU, neonatal intensive care unit; $\mathrm{CH}$, chloral hydrate; PF, propofol; MIDA, midazolam. 
에 영향을 미칠 수 있는 진정 치료 시행 당시 교정나이 및 체중, 진정 약물의 추가 사용을 포함하였다.

\section{4. 이상반응의 종류 및 정의}

뇌 MRI를 위한 진정 후 24 시간 동안의 이상반응 발생 횟수를 수집 하였다. 산소포화도 저하는 말초 산소포화도가 $90 \%$ 미만으로 감소 한 경우, 서맥은 말초 산소포화도의 감소 없이 심박수가 분당 90회 미만으로 감소하는 경우로 정의하였다. 또한 뇌 MRI 시행 후 산소요 구도의 증가에 따른 산소 사용 여부 및 깊은 진정에 의한 호흡 요구 감소 시 기도 삽관, 후두마스크(laryngeal mask airway) 사용 여부에 대해서도 조사하였다. 수유 장애는 수유 후 잔량이 $50 \%$ 이상 남거나 검사 후 반복적 구토를 보이거나 경구 식이에서 경관 식이로 전환되 었을 때로 정의하였다.

Table 1. Demographics of the Enrolled Infants (n=250)

\begin{tabular}{|c|c|c|c|}
\hline Variable & $\begin{array}{c}\text { Period } 1(\mathrm{CH}) \\
(\mathrm{n}=169)\end{array}$ & $\begin{array}{c}\text { Period } 2(P F) \\
(n=81)\end{array}$ & $P$-value \\
\hline \multicolumn{4}{|l|}{ Demographic data } \\
\hline Gestational age (wk) & $27.2 \pm 2.6$ & $27.0 \pm 3.5$ & 0.612 \\
\hline Birth weight (g) & $975.2 \pm 312.2$ & $1,016.4 \pm 508.7$ & 0.503 \\
\hline Male sex & $85(50.3)$ & $45(55.5)$ & 0.499 \\
\hline Small for gestational age & $33(19.5)$ & $16(19.8)$ & 1.000 \\
\hline 1-min Apgar score & $5.4 \pm 1.7$ & $4.6 \pm 1.9$ & 0.004 \\
\hline 5-min Apgar score & $7.3 \pm 1.4$ & $6.7 \pm 1.8$ & 0.009 \\
\hline Cesarean delivery & $139(82.2)$ & $57(70.4)$ & 0.048 \\
\hline Outborn & $24(14.2)$ & $23(28.4)$ & 0.009 \\
\hline Congenital anomaly & $3(1.8)$ & $4(4.9)$ & 0.218 \\
\hline \multicolumn{4}{|l|}{ Neonatal outcome data } \\
\hline IVH grade $\geq 3$ & $16(9.5)$ & $19(23.5)$ & 0.006 \\
\hline PVL & $4(2.4)$ & $9(11.1)$ & 0.006 \\
\hline Seizure & $27(16.3)$ & $13(16.0)$ & 1.000 \\
\hline Moderate to severe BPD & $39(24.2)$ & $16(19.8)$ & 0.516 \\
\hline \multicolumn{4}{|l|}{ MRI-related clinical data } \\
\hline CA at the test (wk) & $37.2 \pm 2.8$ & $38.4 \pm 3.3$ & 0.002 \\
\hline Body weight at the test (g) & $2,068.9 \pm 406.6$ & $2,297.0 \pm 638.6$ & 0.001 \\
\hline Duration of the test (min) & $93.2 \pm 19.5$ & $79.8 \pm 19.1$ & 0.002 \\
\hline $\mathrm{O}_{2}$ use via $\mathrm{NP}$ at the test & $6(3.6)$ & $6(7.4)$ & 0.222 \\
\hline Intubated state at the test & 0 & $1(1.2)$ & 1.000 \\
\hline Final sedation failure* & $2(1.2)$ & 0 & 1.000 \\
\hline Adverse events* & $110(65.1)$ & $51(62.9)$ & 0.887 \\
\hline
\end{tabular}

Values are expressed as number (\%) or mean \pm standard deviation. *Adjusted for gestational age, birth weight, CA, body weight at the MRI test, the presence of IVH and PVL.

Abbreviations: $\mathrm{CH}$, chloral hydrate; $\mathrm{PF}$, propofol; IVH, intraventricular hemorrhage; PVL, periventricular leukomalacia; BPD, bronchopulmonary dysplasia; MRI, magnetic resonance imaging; CA, corrected age; NP, nasal prong.

\section{5. 통계}

SPSS version 25.0 (IBM Co., Armonk, NY, USA)을 사용하였으 며, 두 군 간의 교차분석은 chi-square 또는 Fisher's exact test를 이용 하였으며, 연속변수에는 $t$-test, Mann-Whitney U-test를 사용하였다. 독립 위험인자 규명을 위해 다중 로지스틱 회귀분석을 사용하였다. $P$ 값 0.05 미만인 경우 통계적으로 유의미하다고 정의하였다.

\section{결과}

총 대상자 250 명 중 $\mathrm{CH}$ 를 일차적으로 사용한 1 기는 169 명, $\mathrm{PF}$ 를 사용한 2 기는 81 명이었다. 1 기와 2 기 간의 임신나이, 출생 체중 등 에는 차이가 없었으나, 2 기 환자 중 1 분, 5 분 아프가 점수가 더 낮았 으며, 외부 병원 출생아가 더 많았고, 3 기 이상의 뇌실내출혈 및 낭 성 백질연화증의 빈도가 높았다. 또한, 특징적으로 2기에서 MRI 촬 영 당시의 교정나이가 더 많았으며(37.2주 대 38.4주, $P=0.002$ ), 체 중도 더 높았다 $(2,068 \mathrm{~g}$ 대 $2,297 \mathrm{~g}, P=0.001)$. 반면에 $\mathrm{MRI}$ 촬영 시간 은 93.2분 대 79.8 분으로 1 기에서 더 길었다. 1 기의 169 명 중 90 명 (53\%)은 $\mathrm{CH}$ 초회 투여로 진정에 성공하였으나 77 명(46\%)은 $\mathrm{CH}$ 의 다회 투여 또는 다회 투여 후 미다졸람 사용까지 필요하였다. 또한, 1 기의 2 명( $1 \%)$ 은 진정에 최종적으로 실패하여 MRI 촬영을 시행하 지 못하였다. 반면에 2 기의 81 명은 모두 진정에 성공하여 $\mathrm{MRI}$ 촬영 을 시행 받았다(Table 1).

$\mathrm{MRI}$ 촬영 후 24 시간 이내의 진정 관련 이상반응을 1 기와 2 기 간 에 비교하였을 때, 1 기에서 서맥의 빈도가 의미 있게 증가하였다 (30.2\% 대 14.8\%) (Table 2). 1기의 대상자 중 $\mathrm{CH}$ 를 다회 투여하거 나 다회 투여 후 미다졸람까지 투여한 경우를 $\mathrm{CH}$ 초회 투여한 경우 와 비교했을 때 다회 투여군에서 산소포화도 감소가 의미 있게 증 가하였다(64.6\% 대 44.4\%) (Table 3). 총 대상자를 이상반응 유무 에 따라 나누었을 때 이상반응을 보인 군의 임신나이가 이상반응이 없었던 군에 비해 의미 있게 적었으며(26.8주 대 27.9주, $P=0.004$ ),

Table 2. Comparison of the Adverse Events within 24 Hours after Magnetic Resonance Imaging between Infants in Period 1 and 2

\begin{tabular}{lccc}
\hline Variable & $\begin{array}{r}\text { Period 1 } \\
(\mathrm{n}=169)\end{array}$ & $\begin{array}{c}\text { Period 2 } \\
(\mathrm{n}=81)\end{array}$ & AOR (95\% CI $)$ \\
\hline Increase in desaturation & $91(53.8)$ & $47(58.0)$ & $0.753(0.420-1.352)$ \\
Increase in bradycardia & $51(30.2)$ & $12(14.8)$ & $2.350(1.124-4.911)$ \\
Oxygen supplementation & $30(17.8)$ & $18(22.2)$ & $0.927(0.450-1.911)$ \\
Airway intervention* & 0 & $2(2.5)$ & - \\
Feeding intolerance & $6(3.6)$ & $5(6.2)$ & $0.35(0.07-1.54)$ \\
\hline
\end{tabular}

Values are expressed as number (\%).

*Requiring intubation or laryngeal mask airway.

Abbreviations: AOR, adjusted odds ratio; CI, confidence interval. 
MRI 촬영 당시의 교정나이가 어렸으며(37.3주 대 38.3주, $P=0.023$ ), MRI 촬영 시간 또한 길었다(70.9분 대 64.3분, $P=0.016$ ) (Table 4). 이상반응과 관계된 위험요인을 분석하기 위한 다변량 회귀분석 결 과 출생 당시 임신나이와 MRI 촬영 당시의 교정나이가 많을수록 이 상반응의 위험이 감소하였다(임신나이 교정 오즈비, 0.782; 95\% 신뢰구간, 0.633-0.937) (교정나이 오즈비, 0.827; 95\% 신뢰구간, $0.727-0.940)$.

\section{고찰}

본 연구에서 미숙아 대상으로 MRI 촬영 시 마취과 의사의 모니터 링 하에 $\mathrm{PF}$ 를 사용하여 진정하는 방법은 $\mathrm{CH}$ 를 단독/다회 또는 미다

Table 3. Comparison of the Adverse Events between Infants Who Received Multiple Doses and a Single Dose of $\mathrm{CH}$ in Period 1

\begin{tabular}{|c|c|c|c|}
\hline Variable & $\begin{array}{c}\text { Period } 1 \\
(\mathrm{CH}) \\
\text { multiple* } \\
(\mathrm{n}=79)\end{array}$ & $\begin{array}{c}\text { Period } 1 \\
(\mathrm{CH}) \\
\text { single } \\
(\mathrm{n}=90)\end{array}$ & $\operatorname{AOR}(95 \% \mathrm{CI})$ \\
\hline Increase in desaturation & $51(64.6)$ & $40(44.4)$ & $2.059(1.069-3.968)$ \\
\hline Increase in bradycardia & $29(36.7)$ & $22(24.4)$ & $1.691(0.829-3.450)$ \\
\hline Oxygen supplementation & $19(24.1)$ & $12(13.3)$ & $2.024(0.850-4.819)$ \\
\hline Airway intervention $^{\dagger}$ & 0 & 0 & - \\
\hline Feeding intolerance & $3(3.8)$ & $3(3.3)$ & $0.87(0.170-4.610)$ \\
\hline
\end{tabular}

Values are expressed as number (\%).

${ }^{*} \mathrm{CH}$ multiple doses \pm midazolam; ${ }^{\dagger}$ Requiring intubation or laryngeal mask airway.

Abbreviations: $\mathrm{CH}$, chloral hydrate; $\mathrm{AOR}$, adjusted odds ratio; $\mathrm{CI}$, confidence interval.
졸람 병행하여 비 마취과 의료진에 의해 투여/감시되는 고식적인 방 법에 비해 진정 실패를 줄이고 촬영 후 진정 관련 이상반응을 줄이 는 좋은 방법임을 확인하였다. 진정요법은 신생아에서 뇌 MRI를 시 행할 때 환아의 스트레스를 감소시킬 수 있고, 검사에 방해되는 움 직임을 제한 시켜 검사 진행에 큰 도움을 줄 수 있다. 하지만 의료진 이 잘 훈련되어 있지 않고 시설 장비가 제대로 갖추어져 있지 않다 면 진정요법의 위험성은 언제나 존재하며, 이러한 안전에 대한 우려 에도 불구하고 비 마취과 의료진에 의한 소아 진정은 효율성 및 마 취과 의사 인력의 부족 등의 이유로 아직도 국내외 많은 병원에서 흔히 시행되고 있다. 하지만 마취과 의사가 상주하는 수술실과 달 리 수술실 밖에서의 진정은 경험이 적은 비숙련 의료진에 의해 진정 이 시행되는 경우가 많으며 예기치 못한 사고 발생 시에 적절한 대 처를 하기 어렵다. 본원 소아진정실에는 소아 전담 마취과 의사의 투약 지시에 따라 진정 간호사가 약물을 투여하고 소아 진정 규정에 따라 환자 상태를 약물 투여 직전부터 퇴실 시까지 지속해서 감시하 며 관련된 이상반응을 기록한다. 따라서 비 마취과 의료진이 수행하 는 소아진정요법 보다 더 체계적이고 안전한 환자 관리가 가능하다.

미숙아를 비롯한 소아의 진정을 위해서는 $\mathrm{CH}$, 미다졸람, $\mathrm{PF}$ 등 의 약물이 가장 흔히 사용된다. $\mathrm{CH}$ 는 단기간 소아를 진정시킬 시 에 효과적으로 많이 사용되어 왔지만, 작용 발현 시간을 예측하기 어렵고, 진정에 실패하는 경우가 발생할 수 있다 ${ }^{16,17)}$. 반면PF은 신 생아 및 소아에서 뇌 MRI를 시행할 때 모르핀이나 미다졸람보다 수 면에 이르기까지의 발현 시간이 매우 빠르며, 자발적인 호흡이 가능 하고, 회복시간이 빨라 진정요법으로 적합하다. 다만 중추신경계의 억제가 용량의존적으로 발생할 수 있는데 실제로 뇌 영상검사 시엔 더 적은 용량만을 필요로 하기 때문에 이상반응은 상대적으로 적으 며, 그보다는 검사에 필요한 시간의 절약에 따른 경제적인 효과가 더 크다 ${ }^{18,19)}$. Dalal 등 ${ }^{20)}$ 은 소아 $\mathrm{MRI}$ 촬영 시 $\mathrm{CH}$ 를 사용하는 것이 $\mathrm{PF}$

Table 4. Clinical Characteristics of the Infants with and without Adverse Events

\begin{tabular}{|c|c|c|c|c|}
\hline Variable & Adverse events $(+)(n=161)$ & Adverse events $(-)(n=89)$ & $P$-value & OR $(95 \% \mathrm{CI})$ \\
\hline Gestational age (wk) & $26.8 \pm 2.8$ & $27.9 \pm 3.1$ & 0.004 & $0.782(0.633-0.937)$ \\
\hline Birth weight (g) & $967.8 \pm 376.9$ & $1,026.1 \pm 402.5$ & 0.254 & \\
\hline 1-min Apgar score & $5.2 \pm 1.9$ & $5.0 \pm 1.9$ & 0.411 & \\
\hline 5-min Apgar score & $7.2 \pm 1.6$ & $7.1 \pm 1.7$ & 0.911 & \\
\hline IVH grade $\geq 3$ & $7(4.3)$ & $6(6.7)$ & 0.389 & \\
\hline CA at the MRI test (wk) & $37.3 \pm 2.7$ & $38.3 \pm 3.6$ & 0.023 & $0.827(0.727-0.940)$ \\
\hline Body weight at the MRI test (g) & $2,147.0 \pm 491.7$ & $2,135.3 \pm 528.2$ & 0.864 & \\
\hline Duration of MRI test (min) & $70.9 \pm 21.4$ & $64.3 \pm 18.9$ & 0.016 & $0.981(0.967-0.995)$ \\
\hline
\end{tabular}

Values are expressed as number (\%) or mean \pm standard deviation.

Abbreviations: OR, odds ratio; CI, confidence interval; BPD, bronchopulmonary dysplasia; IVH, intraventricular hemorrhage; CA, corrected age; MRI, magnetic resonance imaging. 
에 비해 심장호흡계 부작용이 더 적게 발생한다고 하였다 $2.9 \%$ 대 $13.6 \%$ ). 본 연구의 결과는 이와 상반되게 PF 연속주입을 한 2 기에서 서맥 발생이 적었는데 이는 마취과 의사의 감독하에 활력징후의 모 니터링이 잘 이루어지고 필요시 적절한 조치가 취해졌다는 점, 이 에 따라 약물의 반복, 과다 투여가 최소화될 수 있었던 점 등에 기인 한 것으로 생각된다. 따라서 본 연구의 결과는 약물 자체의 선택보 다 숙련된 마취 진정 의료진의 감시하에 최소한의 용량으로 약물을 투여하였을 때 진정 실패율이 감소하고, 촬영 후 이상반응의 위험이 감소하며, MRI 촬영 시간도 단축될 수 있음을 시사한다. 반면 2기에 서도 검사 시행 도중 후두마스크, 기관삽관과 같은 기도유지를 위 한 조치가 2예(2.5\%) 발생하였다. 한 예는 임신나이 29주, 1,180 g으 로 출생하여 교정나이 36 주, 체중 $2,000 \mathrm{~g}$ 에 뇌 MRI를 155 분간 시행 하였고, 다른 한 예는 임신나이 30주 5일, $1,310 \mathrm{~g}$ 으로 출생하여 4단 계 뇌실내출혈로 교정나이 36 주, $2,170 \mathrm{~g}$ 에 검사를 75 분간 시행하였 다. 신생아에서 뇌 MRI 검사를 위한 PF 연속주입 시, 예상보다 깊은 진정에서 나타날 수 있는 저환기, 저산소증, 서맥 등의 부작용을 고 려하여 환자 감시, 기도 관리, 호흡 관리, 심폐소생술 등을 잘 숙지한 상태로 능동적 모니터링이 필수적임을 시사한다. 최근에는 신생아 에서 진정제 투여 없이 MRI를 시행하는 방법이 선호되고 있는데, 이 는 충분한 검사시간과 전문 인력 및 시설 필수적이나 궁극적으로 도 입되어야 할 이상적인 방법이겠다. 이 경우에도 마취 진정 의료진의 철저한 감시가 필요할 것이다.

진정 후 이상반응의 발생률은 연구에 따라 차이가 있으며, 진정요 법에서 연령은 이상반응을 일으킬 수 있는 위험인자로 잘 알려져 있 다 ${ }^{21-23)}$. 본 연구에서는 전체 환자의 $64.4 \%$ 에서 이상반응이 나타닜 으며(1기에서 $65.1 \%, 2$ 기에서 $62.9 \%)$, 이는 기존 연구에 비해 상대 적으로 높은 수치였다. 이는 본 연구 대상자의 임신나이 및 검사 당 시 교정나이가 다른 연구와 비교하여 낮아 이상반응의 발생률이 높 게 발생한 것으로 추측된다. 본 연구에서 임신나이가 어릴수록, 촬 영 당시 교정나이가 어릴수록, 또한 촬영 시간이 길수록 이상반응의 위험이 증가하였으며, 특히 여러 변수를 보정 후에도 어린 임신나이 가 이상반응의 위험성을 증가시켰다. 따라서 비록 만삭의 교정나이 (term-equivalent age)에 도달한다고 하더라도 초극소 미숙아와 같 은 어린 임신나이에 출생한 아기들은 진정제 투여에 매우 취약함을 알 수 있다.

본 연구는 단일 연구기관에서 시행한 후향적 연구로 대상자 수가 적고, $\mathrm{MRI}$ 실에서 검사 시행 시간 동안 활력징후 및 호흡기계의 억제 에 대한 정확한 정보가 부족했다는 점에서 한계를 지닌다. 또한 모 든 미숙아에서 일률적으로 뇌 MRI를 시행했던 1기와 달리 2기에서 는 뇌실내출혈, 저산소성 허혈성뇌병증 등 뇌질환을 가진 미숙아에 게서 선택적으로 시행했으므로 두 기간의 대상자의 임상적 특징이 달랐던 점도 한계로 생각할 수 있다. 또한 2기의 MRI 적응증은 뇌 질환 의심자로 한정되었지만 뇌질환 여부는 담당 신생아 의사의 임
상적 판단에 의거하였다. 따라서 1 기와 2 기 대상자 간의 여러 임상 적 차이가 편향으로 작용할 가능성을 배제할 수 없었으며 이를 다변 량 회귀분석을 통해 보완하려 하였다. 하지만 통제하지 못한 변수 로 인한 편향이 존재할 가능성이 있으며 이는 본 연구의 한계로 지 적할 수 있다.

결론적으로 본 연구 결과, 미숙아에서의 뇌 MRI 촬영 시 PF 연속 주입을 통한 진정은 기존의 $\mathrm{CH}$ 단독/다회 투여 및 미다졸람 병행 투 여 등의 고식적 진정에 비해 이상반응이 적고 검사 시간을 단축시킬 수 있었다. 단 이는 약제의 우월성보다 소아 마취 진정 전문 의료진 의 감시와 개입에 의한 것으로 판단된다. 임신나이와 교정나이가 어 릴수록, 약물의 다회 투여가 될수록 이상반응의 위험이 증가하므로 더욱 철저한 관찰이 필요하겠다.

\section{ARTICLE INFORMATION}

\section{Ethical statement}

This study was approved by the Institutional Review Board of Samsung Medical Center (IRB No. 2020-04-144). Written in formed consent by the patients was waived due to a retrospective nature of our study.

\section{Conflicts of interest}

No potential conflict of interest relevant to this article was reported.

\section{Author contributions}

Conception or design: Y.S.H., S.Y.A., S.I.S., Y.S.C.

Acquisition, analysis, or interpretation of data: Y.S.H., H.H.K., H.S.K., M.S.Y.

Drafting the work or revising: Y.S.H. S.I.S., W.S.P.

Final approval of the manuscript: S.I.S.

\section{ORCID}

Yea Seul Han https://orcid.org/0000-0002-4074-2936

Se In Sung https://orcid.org/0000-0002-8717-6142

\section{Acknowledgments}

None 


\section{REFERENCES}

1. Slaughter LA, Bonfante-Mejia E, Hintz SR, Dvorchik I, Parikh NA. Early conventional MRI for prediction of neurodevelop mental impairment in extremely-low-birth-weight infants. Neonatology 2016;110:47-54.

2. Pennock JM. Patient preparation, safety and hazards in imaging infants and children. In: Rutherford M, editor. MRI of the neonatal brain. London: W.B. Saunders, 2002:3-15.

3. Hall RW, Shbarou RM. Drugs of choice for sedation and analgesia in the neonatal ICU. Clin Perinatol 2009;36:15-26.

4. Litman RS, Soin K, Salam A. Chloral hydrate sedation in term and preterm infants: an analysis of efficacy and complications. Anesth Analg 2010;110:739-46.

5. Finnemore A, Toulmin H, Merchant N, Arichi T, Tusor N, Cox D, et al. Chloral hydrate sedation for magnetic resonance imaging in newborn infants. Paediatr Anaesth 2014;24:190-5.

6. Pershad J, Palmisano P, Nichols M. Chloral hydrate: the good and the bad. Pediatr Emerg Care 1999;15:432-5.

7. Rigby-Jones AE, Nolan JA, Priston MJ, Wright PM, Sneyd JR, Wolf AR. Pharmacokinetics of propofol infusions in critically ill neonates, infants, and children in an intensive care unit. Anesthesiology 2002;97:1393-400.

8. Machata AM, Willschke H, Kabon B, Kettner SC, Marhofer P. Propofol-based sedation regimen for infants and children undergoing ambulatory magnetic resonance imaging. $\mathrm{Br} \mathrm{J}$ Anaesth 2008;101:239-43.

9. Pershad J, Wan J, Anghelescu DL. Comparison of propofol with pentobarbital/midazolam/fentanyl sedation for magnetic re sonance imaging of the brain in children. Pediatrics 2007;120: e629-36.

10. Arthurs OJ, Sury M. Anaesthesia or sedation for paediatric MRI: advantages and disadvantages. Curr Opin Anaesthesiol 2013; 26:489-94.

11. Allegaert K, Daniels H, Naulaers G, Tibboel D, Devlieger H. Pharmacodynamics of chloral hydrate in former preterm in fants. Eur J Pediatr 2005;164:403-7.

12. Smallman B. Pediatric sedation: can it be safely performed by non-anesthesiologists? Curr Opin Anaesthesiol 2002;15:455-9.

13. Chiaretti A, Benini F, Pierri F, Vecchiato K, Ronfani L, Agosto C, et al. Safety and efficacy of propofol administered by paediatricians during procedural sedation in children. Acta Paediatr 2014;103:182-7.

14. Krauss BS, Krauss BA, Green SM. Procedural sedation and analgesia in children. N Engl J Med 2014;371:91.

15. Cook-Sather SD, Litman RS. Modern fasting guidelines in children. Best Pract Res Clin Anaesthesiol 2006;20:471-81.

16. Choi YS, Son YJ, Song ES, Cho YK, Kim YO, Kim CJ, et al. Effect of chloral hydrate used for pediatric sedation. J Korean Child Neurol Soc 2008;16:78-85.

17. Delgado J, Toro R, Rascovsky S, Arango A, Angel GJ, Calvo V, et al. Chloral hydrate in pediatric magnetic resonance imaging: evaluation of a 10-year sedation experience administered by radiologists. Pediatr Radiol 2015;45:108-14.

18. Allegaert $\mathrm{K}$. The clinical pharmacology of short acting analgosedatives in neonates. Curr Clin Pharmacol 2011;6:222-6.

19. Welzing L, Kribs A, Eifinger F, Huenseler C, Oberthuer A, Roth B. Propofol as an induction agent for endotracheal intubation can cause significant arterial hypotension in preterm neonates. Paediatr Anaesth 2010;20:605-11.

20. Dalal PG, Murray D, Cox T, McAllister J, Snider R. Sedation and anesthesia protocols used for magnetic resonance imaging studies in infants: provider and pharmacologic considerations. Anesth Analg 2006;103:863-8.

21. Cravero JP, Blike GT, Beach M, Gallagher SM, Hertzog JH, Havidich JE, et al. Incidence and nature of adverse events during pediatric sedation/anesthesia for procedures outside the operating room: report from the pediatric sedation research consortium. Pediatrics 2006;118:1087-96.

22. Allegaert K, Peeters MY, Verbesselt R, Tibboel D, Naulaers G, de Hoon JN, et al. Inter-individual variability in propofol pharmacokinetics in preterm and term neonates. Br J Anaesth 2007; 99:864-70.

23. Havidich JE, Beach M, Dierdorf SF, Onega T, Suresh G, Cravero JP. Preterm versus term children: analysis of sedation/ane sthesia adverse events and longitudinal risk. Pediatrics 2016; 137:e20150463. 